\title{
Personalized and Real-Time Data Oriented Disease Inferring System
}

\author{
Rasla Azeez ${ }^{1}$, Anju C R ${ }^{2}$ \\ ${ }^{1,2}$ KMCT College of Engineering, Calicut, Kerala, India
}

\begin{abstract}
As we all know health status is an concerning factor by each human. An important problem of current Web search is that search queries are usually short and not enough for knowledge inferring, and thus are not good enough for specifying the precise user need. Automatic disease inference is of importance to reduce the distance between knowledge to be inferred and needs of the user. This knowledge mining is useful especially for community-based health services due to the vocabulary gap, incomplete information, correlated medical concepts, and limited high quality training samples. There are many online and offline methods are there to getting the information requested by the health seeker. Methods like questionnaire, deep learning are used as inferring methods. Here the sparse deep learning algorithm is used as the data mining technique. In this paper, here first perform a health seeker study on the information needs of health seekers in terms of queries. Some attributes used are raw features, signatures, medical attributes etc. Deep learning refers to the depth wise analysis of the raw features and their signatures as input nodes in one layer and hidden nodes in the next layers of the learning architecture.. Because of the deepest and alternative repeating of these features, our architecture a sparsely connected deep learning technique maintained with three hidden layers. That is, it learns the internal relations between the data collected and several layers. Abstract signature mining will produces the deepest knowledge about enquiry process towards health seeking. It will give out specific tasks with fine-tuning, pre-tuning etc. Several experiments on day today dataset given by online doctors show the significant performance and importance of this disease inference. Here along with sparse deep learning, real-time health data, user history and bidirectional querying makes the system much useful than the existing system.
\end{abstract}

Keywords: SVM (Support Vector Machine), Sparse deep learning, Classifiers, Querying, Signature mining.

\section{Introduction}

The main aim of this paper is to line up and emphasize the importance of exploratory analytics that are commensurate with human capabilities and constraints along with updates of existing exploratory data.The increasing in the development of each and every countries, expenditure of healthcare and emergence in computer technologies all are the major reasons for the innovation to the automatic health seeking system. Analyzing a survey it is easy to understand that most of the people are utilizes the availability of emerging technologies such as computers, journals, magazines, internet technologies etc. The Knowledge mining in health records is a key aspect for improved clinical decision making, and patient management, population management etc. Increased internet technologies in health seeking promises towards better data aggregation, automated requesting and updating, and clinical development etc. The large number of information they capture over time pose challenges not only for medical practitioners, but also for the information analysis by machines and local users they are intended to get satisfied with their need.

In this realm we present a sparse deep learning architecture that discovers complex medical attributes, medical signatures and its corresponding matching patterns, which are easily understandable by users. Here enhance this framework based on users need and available prior data. The analysis study pointing towards several aspects of users need.

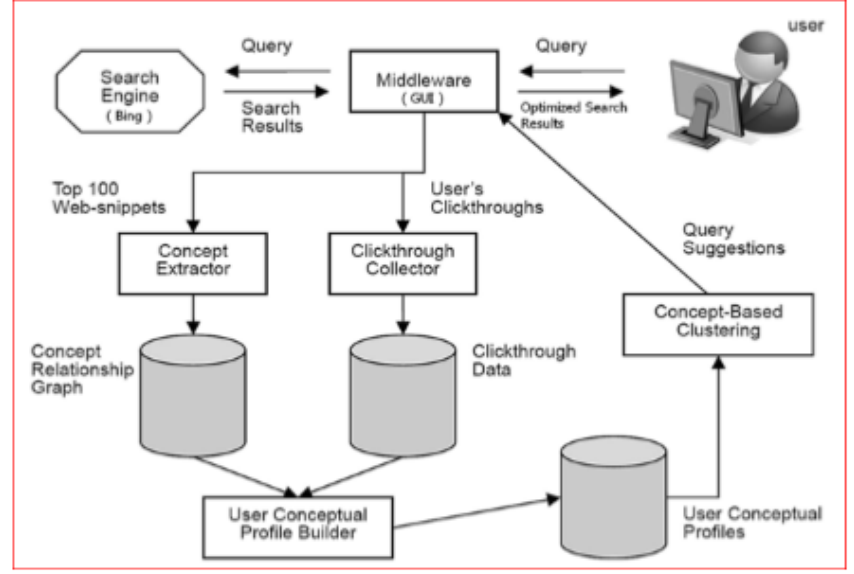

Figure 1: Disease Inferring Architecture

The above disease inferring system(DIS),illustrating about how an user or health seeker can be meet his health requirements.WebMD1, health tap, MedlinePlus2 are the typical examples of health data providers. Community-based health services, such as HealthTap3 and HaoDF4 are examples of community based seeking. There are some interactive platforms for the seekers. They can anonymously ask health-oriented questions and at that time doctors provide the knowledgeable and trustworthy answers. Typical example can be explained as user can ask question regarding about their health need towards the system. Community based health service is very time consuming for health seekers to get their posted questions resolved in a particular period of time. While considering any other online methods we can see that doctors are compelled to finish replies for number of problems that are requested by users because all it is an important factor to identify solution for the above so here develop automatic and comprehensive wellness systems that

Volume 5 Issue 6, June 2016 www.ijsr.net 


\section{International Journal of Science and Research (IJSR) \\ ISSN (Online): 2319-7064}

Index Copernicus Value (2013): 6.14 | Impact Factor (2015): 6.391

can instantly answer all-round questions of health seekers and alleviate the doctors' workload. Along with the application of a bidirectional querying will improve the performance of the system in a greater amount. The resolving time could vary from hours to days some may be more than these. Next limitation is that doctors having to cope with an everexpanding workload, which leads to reduction in efficiency. The Support Vector Machine (SVM) is the classifier used to classify the data in a sparse deep learning manner.

They are very accurate and generalize well to a wide range of operations. To understand this just considers an SVM used by a bank to determine to whom they will loan money. If a user's loan application is rejected and they would like to getting the information about why it is rejected,Support Vector Machine is a best classifier and we used it here, Support vector machines (SVMs) are a well- known supervised learning technique for performing binary classification in data classification. The resolving time could vary from hours to days some may be more than these. Next limitation is that doctors having to cope with an everexpanding workload, which leads to reduction in efficiency. here it is not very useful to only be able to say that the algorithm came back with a number lower than some required threshold. There will be notification, and that would be much more satisfactory to the customer to be able to tell them that they were rejection of credit because their income is too low and they have six outstanding loans. Since support vector machines are "black-box" classifiers, the decisions they make are not always easily explainable. Because of this we mean that the model produced does not automatically provide any helpful intuitive reasons about why a particular point is classified into a class rather than another classifier.. The Decision trees can provide a structure that is much more easily interpreted, but unable to interpret always to achieve results as accurate as those produced by an SVM. In this innovation SVM is replaced by Boolean ranking so that it can produce a better ranking and result optimization than SVM.

\section{Literature Survey}

Because of the growing aged population coupled with lack of medical services and healthcare services in most of the developing countries, the traditional health- care system meets challenging problems caused by its high operating cost and un scalability. Compared to the conventional healthcare system, there is a need of more accurate and easy to access system to improve quality of health care services. The following are the some of the parameters we needed to be improved for the health care services. The existing system mainly focuses on healthcare service in a physiological and psychological aspect with the following two undesirable features etc. They are improving the quality of medical service, Improving the utilization of medical helps and care by enabling remote medical services, and supporting the development of the health industry. The existing literatures [9],[5] and [8],[6] are explaining some discriminant features. Where the basic methodology used in this advanced health care is explained by support Vector Machine
classifier[1].The literatures[3],[4],[2],[7] following some earlier methods of health services.

David Barbella [1] proposed a system the where SVM or the Support vector machines are a valuable and useful tool for making classifications. But their black-box nature means that they lack the natural explanatory value that many other classifiers possess. In the first, we report the support vectors most touching in the final classification for a particular test location. Next we determine which features of that test location would need to be changed in order to be placed on the separating surface between the two classifications. Another technique is called "border classification." In order to explaining these explanatory techniques, we also present a free-for-download software tool that enables users to visualize these insights graphically. In other way, many useful and great famous websites have shown recent success in explaining recommendations based on nature of other users. Accepting by these ideas, we recommend two novel methods for providing insight into local classifications produced by a SVM.

F. Wang [2] proposed a temporal knowledge representation and learning framework to perform large scale temporal signature mining of longitudinal heterogeneous event data occurrences. Novel stochastic optimization architecture performs large-scale incremental learning of group-specific temporal event signatures. It evaluates the framework on synthetic data and on an electronic health record dataset and its manipulation. Here present a doubly constrained convolutional sparse coding architecture that learns interpretable and shift-invariant latent temporal event signatures.

N. Lee [3] established the knowledge discovery in electronic health records (EHRs) as a central aspect for improved clinical decision making, prognosis, health data management and patient management. Where EHRs show great promise towards better data integration, automated access, and clinical workflow improvement, the detailed information they collect over time face challenges not only for medical practitioners, but also for the information analysis by machines.

The aim of this is to motivate the importance of exploratory analytics that are commensurate with human capabilities and constraints to be meet. Here this architecture on synthetic data and on EHRs together with an extensive validation involving many computed latent factor models. While using this realm we present a novel temporal event matrix representation and learning architecture that discovers complex latent event patterns, which are easily interpretable by human beings.

In Amitpande [4] SSIEEE, used built-in smartphone sensors (accelerometer and barometer sensor), sampled at low frequency, to accurately estimate Energy Expenditure. Here also using a barometer sensor, in addition to an accelerometer sensor, greatly increases the accuracy of Energy Expenditure estimation. 


\section{International Journal of Science and Research (IJSR) \\ ISSN (Online): 2319-7064}

Index Copernicus Value (2013): 6.14 | Impact Factor (2015): 6.391

Lejun Gong [5] proposed a system where latest disease holding genes could be detected. Understanding the hand of genetics in diseases is one of the most important and greedy tasks in the post genome era. Genetic association analysis and diversions has proven to be a successful tool to enhance the knowledge about genetic risk components to a variety of complex diseases. Measuring the functional similarity between known disease susceptibility genes and unknown genes is to predict new disease susceptibility genes.

There are wide applications of computational methods in discovering gene responsible for human disease. Here propose an approach to prioritize disease susceptibility genes using LSM/SVD. Measuring the functional similarity between known disease susceptibility genes and unknown genes is to predict new disease susceptibility genes. It could discover again latest disease holding genes. This new approach of disease gene prioritization could discover new disease affecting genes.

M.Shouman[6] established the availability of large amounts of medical data that leads to the need for powerful data analysis tools to extract useful knowledge for finding a particular need towards health. While using single data mining technique in the diagnosis of a disease especially in heart, has been comprehensively investigated showing acceptable levels of correctness.

Y. Bengio[7] proposed a representation learning that evaluate the learning process in a better way. The performance of machine learning techniques is heavily dependent on the choice of data representation (or features) on which they are applied. This is important but labour intensive and highlights the weakness of current learning techniques.Its disability to extract and organize the discriminative information from the data. Feature engineering is a way to take advantage of human unawareness and prior knowledge to compensate for that disability. To expand the scope and ease of applicability of machine learning, it would be highly desirable to make learning algorithms less dependent on feature engineering so that novel applications could be constructed faster, and more importantly, to make progress toward artificial intelligence (AI).Because of that reason, much of the actual effort in deploying machine learning algorithms goes into the design of pre-processing pipelines and data transformations that result in a representation of the data that can support effective machine learning techniques. An Artificial Intelligence must fundamentally understand the world around us, and we go straight forward that this can only be achieved if it can learn to identify and disentangle the underlying explanatory factors hidden in the observed of low-level sensory data for any learning scheme.

In Olumurejiwa[8], the Medical equipment in developing world health facilities is largely insufficient and inappropriate, resulting in reduced capacity for hospitals and clinics, suboptimal outcomes for patients, and wasted money for donors and investors.

In Gerberding[9] public health is an important factor for the wellness of a country, so public health surveillance is the ongoing, systematic collection, analysis, interpretation, and dissemination of data about a health-related event for use in public health action to reduce morbidity and mortality and to improve health status. The foundation of communicable disease inference in the United States is the state and local application of the reportable disease inference system known as the National Fortifiable Disease Surveillance System (NNDSS), which holds the listing of diseases and laboratory findings of public health interest, the publication of case definitions for their surveillance, and a system for passing case reports from local to state to $\mathrm{CDC}$ and that will perform an efficient health wellness system.

The valuation should be shortened to convey the strengths and weaknesses of the system under scrutiny. Shortening and reporting evaluation findings should facilitate the comparison of systems for those making decisions about new or existing surveillance methods. An Institute of Medicine study concluded that although innovative surveillance methods might be increasingly useful in the detection and monitoring of outbreaks, a balance is needed between strengthening proven approaches (e.g., diagnosis of infectious illness and strengthening the difference between clinical facilities providers and health controllers) and the exploration and evaluation of new methods.

Min Chen [10] proposed a system called AIWAC to reduce the heavy burden from rapidly growing demands of healthcare service. A wearable computing-assisted healthcare has been proposed for health monitoring and remote medical care services. In AIWAC it supports Hybrid emotional data analysis, which supports computation-intensive analysis of various emotional data from CPS-Spaces, Dynamic resource perception and allocation by which user's status can be analyzed, which provides users with real-time, available, and effective and affective interaction. At last an AIWAC test bed is used for emotion-aware application, based on a robot has been presented.

Liqiang Nie[11] established a system in which disease is inferred from Health-Related Questions via Sparse Deep Learning technique is an efficient technique to identify diseases, or to monitor the health status have greatly hindered the performance of classic shallow machine learning approaches.

\section{Modules of Proposed System}

These system is composed of four main modules, they are concept extraction module, sparse deep learning, personalized data creation, real-time data embedding module. The concept relationship graph is first derived without taking user click throughs into account. Intuitively, the graph shows the possible concept space arising from users queries. The concept space, in general, covers more than what the user actually wants. For example, when the user searches for the query apple, the concept space derived from the web-snippets contains concepts such as ipod, iphone, and recipe. If the user is indeed interested in the concept recipe and clicks on pages containing the concept recipe, the clickthroughs should gradually favor the concept recipe and its neighborhood (by assigning higher weights to the nodes), but the weights of the 


\section{International Journal of Science and Research (IJSR) \\ ISSN (Online): 2319-7064}

Index Copernicus Value (2013): 6.14 | Impact Factor (2015): 6.391

unrelated concepts such as iphone, ipod, and their neighborhood should remain zero.

Sparse deep learning will processes the query in a deep way like layer by layer manner. Ranking procedure is done by calculating the occurrences of signatures or medical terms in the data set provided. Personalized data is created by using personal information, click through data and by previously detected diseases(if there is any previously detected disease). Real-time data is embedded to the system by using sensors which can detect the user's body status like blood pressure or (heart rate, body temperature) etc.
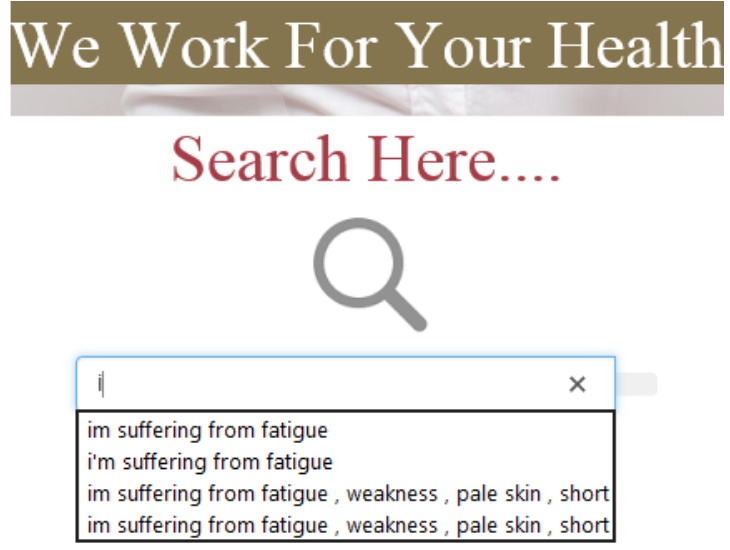

Figure 2: Disease inferring platform

Internet on things, can provide such devices, also it is able to manually compose a sensing device by using sensing unit along with connection sub units.

\section{Conclusion and future scope}

This paper first concentrated towards user study to analyze the health seeker needs. This provides the insights of community-based health services. It then presented a sparsely connected deep learning scheme that is able to infer the possible diseases based on the questions of health seekers. Then Boolean ranking scheme enables the simplification of the generated disease list to a minimum set. It permits unsupervised feature learning from other wide range of disease types. Bidirectional querying is an important step that make some major degree of positive role here, along with click-through data with personalized data will it much better performing platform for health seekers. In future works there is a way to concentrate for some more improvements is multiple bidirectional querying for a single disease.

\section{References}

[1] David Barbella1, Sami Benzaid2, Janara Christensen3,Bret Jackson4, X. Victor Qin "Understanding Support Vector Machine Classifications via a Recommender System-Like Approach"

[2] F. Wang, N. Lee, J. Hu, J. Sun, S. Ebadollah, and A. Laine, "A framework for mining signatures from event sequences and its applications in healthcare data," IEEE Transactions on Pattern Analysis and Machine Intelligence, 2013.
[3] F. Wang, N. Lee, J. Hu, J. Sun, and S. Ebadollahi, "Towards heterogeneous temporal clinical event pattern discovery: A convolutional approach," in The ACM SIGKDD Conference on Knowledge Discovery and Data Mining, 2012.

[4] Amitpande, JindanZhu1, Aveek K. Das1, Yunze Zeng1, Prasanth Mohapatra1, (Fellow, IEEE), And Jay J. Han “ Using Smartphone Sensors for Improving Energy Expenditure Estimation"

[5] Lejun Gong*, Ronggen Yang, Qin Yan, and Xiao Sun, “ Prioritization of Disease Susceptibility Genes Using LSM/SVD"

[6] M.Shouman, T. Turner, and R. Stocker, "Using decision tree for diagnosing heart disease patients," in

Proceedings of the Australasian Data Mining Conference, 2011.

[7] Y. Bengio, A. Courville, and P. Vincent, "Representation learning: A review and new perspectives," IEEE Transactions on Pattern Analysis and Machine Intelligence, 2013.

[8] Refinement of the Facility-Level Medical Technology Score to Reflect Key Disease Response Capacity and Personnel Availability, Olumurejiwa A. Fatunde (Student Member, IEEE)1, And Timothy W. KOTIN (Student Member, IEEE).

[9] Gerberding, M.D., M.P.H.“'Centers for Disease Control and Prevention" Julie L.

[10] AIWAC: Affective Interaction Through Wearable Computing And Cloud Technology, Min Chen, Yin Zhang, Yong Li, Mohammad Mehedi Hassan, And Atif Alamri

[11] Liqiang Nie, Meng Wang, Luming Zhang, Shuicheng Yan, Member, IEEE, Bo Zhang, Senior Member, IEEE, Tat-Seng Chua, Senior Member, IEEE "Disease Inference from Health-Related Questions via Sparse Deep Learning".

\section{Author Profile}

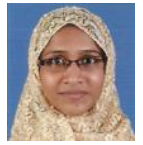

Rasla Azeez is pursuing her M.Tech degree in Computer Science and Engineering from KMCT College of Engineering, Calicut University. She obtained her B.Tech Degree in Computer Science and Engineering from KMCT College of Engineering, in 2013. 